\title{
Subacute effects of edible film from modified sago starch in rats.
}

\begin{abstract}
The effects of edible film from modified sago starch were determined on female SpragueDawley rats where the aim was to develop a basic guideline for safe use of edible films as food packaging. Rats were fed with $2 \mathrm{~g} \mathrm{~kg}-1$ (low dose) and $5 \mathrm{~g} \mathrm{~kg}-1$ (high dose) of body weight edible film for 28 days and the control rats only received normal rat pellet. The study showed that repeated administration of high dose edible film to rats did produce significant change in the liver function (total bilirubin) and renal function parameters (creatinine). Other metabolic parameters tested were: aspartate transaminase, alanine transaminase and alkaline phosphatase, blood lipid profile; cholesterol, high-density lipoprotein, low-density lipoprotein and triglyceride, total protein and glucose concentrations, however, they were not significantly changed with respect to control animals. In conclusion, edible film from modified sago starch at $2 \mathrm{~g} \mathrm{~kg}-1$ is less likely to develop toxicity as observed in the sub-acute toxicity study.
\end{abstract}

Keyword: Edible film; Sago; Starch; Sub-acute; Toxicity. 